\title{
A variabilidade espacial das famílias de Coleoptera (Insecta) entre fragmentos de Floresta Ombrófila Mista Montana (Bioma Araucária) e plantação de Pinus elliottii Engelmann, no Parque Ecológico Vivat Floresta, Tijucas do Sul, Paraná, Brasil ${ }^{1}$
}

\author{
Norma G. Ganho ${ }^{2} \&$ Renato C. Marinoni ${ }^{3}$ \\ ${ }^{1}$ Contribuição número 1659 do Departamento de Zoologia, Universidade Federal do Paraná. \\ 2 Bolsista do CNPq. E-mail: jomario@uol.com.br \\ ${ }^{3}$ Departamento de Zoologia, Universidade Federal do Paraná. Caixa Postal 19020, 81531-980 Curitiba, Paraná, Brasil. \\ Bolsista do CNPq. E-mail: rcmari@ufpr.br
}

\begin{abstract}
Spatial variability of Coleoptera (Insecta) families between a Montane Ombrophilous Mixed Forest (Bioma Araucaria) and Pinus elliottii Engelmann plantation fragments, in the Parque Ecológico Vivat Floresta, Tijucas do Sul, Paraná, Brazil. An important question for biodiversity is what is the impact of monocultures of exotic forest trees on native fauna, especially insects. Coleoptera have been shown to be sensitive to smallscale variations in forest floristic and structure. To test this idea, a comparison of families of Coleoptera was made between a natural forest and a monoculture of Pinus elliottii Engelmann at the Vivat Floresta Ecological Park, Tijucas do Sul, Paraná, in southern Brazil. A one-year inventory was carried out from August 2004 to July 2005 with three malaise traps in each forest type, to compare, in this first preliminary study, family richness and abundance. A total of 12,397 insects in 57 families were collected. Abundance was greatest in the natural forest, while family richness was greatest at the border of the two forest types. As commonly found, studies on comparative abundance composition of areas including only dominant families involved in the first $60 \%$ of total abundance in any given area show the same trends as studies that include total abundance of all the families. In the pine monoculture, the dominant families were, in order of abundance, Cerambycidae, Staphylinidae, Curculionidae, Nitidulidae, Lampyridae, Scolytidae and Chrysomelidae. In the natural forest, the order of dominance was Chrysomelidae, Cerambycidae, Curculionidae, Lampyridae, Nitidulidae and Staphylinidae.

KEY WORDS. Araucaria forest; exotic pine plantation; family composition; family constancy; malaise trap.
\end{abstract}

RESUMO. Várias questões sobre a biodiversidade vêm sendo levantadas nas últimas décadas. Uma delas é o papel que as plantações florestais exóticas monoculturais desempenham na manutenção da fauna nativa, principalmente de insetos. Estudos têm demonstrado que os Coleoptera são sensíveis a variações florísticas e estruturais, em pequena escala espacial. Para analisar as possíveis diferenças na diversidade de Coleoptera entre um ambiente de floresta natural e uma plantação florestal exótica monocultural foi desenvolvido um inventário no Parque Ecológico Vivat Floresta, Tijucas do Sul, Paraná. Para tanto, durante 52 semanas (agosto de 2004 a julho de 2005), seis armadilhas malaise foram dispostas ao longo de um transecto através de dois ambientes adjacentes, com diferentes condições florísticas: três em fragmento da floresta natural (Floresta Ombrófila Mista) e três na plantação de Pinus elliottii exótico. Neste primeiro estudo, as comunidades de Coleoptera foram analisadas com base na abundância e na riqueza das famílias. Foram coletados 12397 exemplares de 57 famílias. A abundância foi maior na floresta natural, decrescendo do interior desta para o interior da plantação de pinus. O ecótono - borda da floresta natural/borda da plantação de pinus - foi o mais rico em famílias. Como observado em inventários de outras localidades, os estudos apoiados em dados das famílias que se incluem nos primeiros $60 \%$ da abundância total de cada área, mostram os mesmos resultados quando são aplicados os dados de todas as famílias. Na plantação de pinus as famílias dominantes foram, pela ordem: Cerambycidae, Staphylinidae, Curculionidae, Nitidulidae, Lampyridae, Scolytidae, Chrysomelidae; na floresta natural: Chrysomelidae, Cerambycidae, Curculionidae, Lampyridae, Nitidulidae, Staphylinidae.

PALAVRAS-CHAVE. Composição de famílias; famílias dominantes; plantação exótica de pinus; armadilha malaise.

Revista Brasileira de Zoologia 23 (4): 1159-1167, dezembro 2006 
O aumento da consciência sobre a importância da biodiversidade criou um impasse entre as necessidades crescentes de obtenção de matéria prima extraída da natureza e os efeitos nocivos resultantes da exploração destes recursos naturais. Neste cenário, destacam-se os ecossistemas florestais, que nas últimas décadas tem passado por um processo crescente de devastação em diferentes regiões.

O processo de desmatamento de áreas naturais atingiu proporções significativas no Brasil. No Paraná isto ocorreu de maneira indiscriminada, principalmente a partir da segunda metade do século passado, resultando numa cobertura vegetal reduzida a cerca de $9 \%$ da original (FundAÇÃo SOS MATA ATLÂNTICA 1998). Depois de destruídas as florestas naturais, as plantações florestais foram cultivadas sem compromisso com a biodiversidade. No entanto, estudos tornam cada vez mais evidente que ela tem implicações diretas nas plantações florestais e em seu manejo (HartLey 2002, Altieri et al. 2003).

As considerações contrárias à plantação florestal monocultural advertem que estas são potencialmente negativas devido à redução da biodiversidade, à invasão de espécies exóticas, além de causar aumento da vulnerabilidade a pestes (RUSSEL 1989, Engelmark et al. 2001).

Por outro lado, alguns estudos indicam casos de comunidades animais em plantações que são similares às das áreas naturais, em que pode até ocorrer um aumento de riqueza. Tal foi observado por Brockie (apud CARnus et al. 2003) no levantamento de pássaros na Nova Zelândia; por Hum PHREY et al. (1999, 2000) avaliando comunidades de invertebrados e fungos na Inglaterra; GANHO \& MARINONI (2005) e MARINONI \& GANHO (2006), com besouros em Ponta Grossa, Paraná.

Estudos da diversidade de Coleoptera em plantações de florestas exóticas, por vezes comparada à fauna de floresta natural dos ambientes onde estavam inseridas, foram os de HUTCHESON \& Jones (1999) e CHung et al. (2000).

No Estado do Paraná, trabal ho gerado por MARINonı \& DUTRA (1997), envolveu o estudo da riqueza e diversidade de famílias de Coleoptera, produto de inventário realizado em oito diferentes local idades, em áreas com diferentes níveis de conservação. GANHO \& MARINONI (2003) publicaram um primeiro trabalho sobre os besouros do Parque Estadual de Vila Velha, ao qual se seguiram outros tratando das diversidadesinventarial ediferencial em cinco ambientes com diferentes características da flora nativa.

Dando seqüência a estudos da fauna de Coleoptera, foi feito um levantamento no Vivat Floresta Sistemas Ecológicos, no município de Tijucas do Sul, Paraná, envolvendo um fragmento defloresta natural em regeneração de Floresta Ombrófila Mista e uma plantação de espécie florestal exótica, Pinus elliottii. Neste trabalho inicial, é estabel ecida uma comparação entre as composições de famílias inventariadas em cada uma das duas áreas florestadas. Buscou-se acrescer informações sobre a viabilidade do uso dos Coleoptera como indicadores ambientais, e verificar a possibilidade do emprego apenas das famílias mais abundantes para que se atinjam tais objetivos.

\section{MATERIAL E MÉTODOS}

Este projeto de inventariamento (PROVIVAT) foi desenvolvido no Parque Ecológico Vivat Floresta (738,99 ha), município de Tijucas do Sul, Paraná, uma das áreas que compõe o Vivat Floresta Sistemas Ecológicos. Esta área (3505 ha) situa-se geograficamente entre $25^{\circ} 45^{\prime}-26^{\circ} 00^{\prime} \mathrm{S}$ e $49^{\circ} 20^{\prime}-499^{\circ} 05^{\prime} \mathrm{W}$, em uma altitude variando entre 850 e 1350 m s.n.m., sobre o Primeiro Planalto Paranaense, na região de domínio da Floresta Atlântica (sensu latu). A área geomorfologicamente originária do mesmo sistema estrutural da Serra do Mar, constituída, em geral, por blocos de rochas cristalinas suaves a fortemente dobrados, sustenta uma paisagem de topografia suave a fortemente ondulada, com colinas de topos amplos e de altitudes relativamente uniformes (VIVAT 2003).

O clima predominante, segundo o método de Köppen, é do tipo Cfb: subtropical úmido, mesotérmico, de verões frescos e com ocorrência de geadas severas e freqüentes. A temperatura média dos meses mais quentes é inferior a $22^{\circ} \mathrm{C}$ e a dos meses mais frios é inferior a $18^{\circ} \mathrm{C}$. No inverno ocorrem fortes geadas, entre cinco e 20 por ano, ou mais em anos atípicos com invernos mais severos.

Predomina a formação da Floresta Ombrófila Mista (Floresta com Araucária), nos patamares Montano e Aluvial. A vegetação da região expressa um mosaico entre alguns relictos primários, que ainda abrigam espécies vegetais ameaçadas de extinção no Estado do Paraná como a imbuia (Ocotea porosa (Nees)) e o sassafrás (Ocotea odorifera (Vellozo)), formações secundárias em diferentes estágios de desenvolvimento e reflorestamentos de Pinus spp., de Eucalyptus spp. e de pinheiro do Paraná (Araucaria angustifolia (Bertoloni) Kuntze), bem como pequenas áreas ocupadas por pastagens.

A maior parte da vegetação, com exceção dos patamares mais el evados, encontra-se bastante al terada em função da agricultura intensiva e da exploração madeireira não planejada, que perdurou até 1974.

\section{Pontos de coleta}

Para o inventariamento da fauna entomológica foram amostradas duas áreas adjacentes; uma delas coberta por Floresta Ombrófila Mista Montana (cerca de 130 ha) e outra por plantação homogênea de Pinus elliottii (cerca de 104 ha).

A parte de Floresta Ombrófila Mista, até cerca de 20 anos atrás, era submetida a desbastes sucessivos de pequenas áreas para cultivo de subsistência, constituindo um mosaico com diferentes níveis de sucessão vegetal; o tal hão com Pinus elliottii foi plantado há 22 anos e não foi submetido a desbastes. 0 sub-bosque e a vegetação do solo nos pontos de coleta são descritos adiante.

Foram utilizadas seis armadilhas malaise (Townes 1972): três na Floresta Ombrófila Mista e três na plantação de Pinus, dispostas em um transecto de acordo com técnica proposta por HutCheson (1990) e Hutcheson \& Jones (1999). Os pontos de coleta foram instalados a diferentes distâncias da borda de con- 
tato para o interior das duas áreas. A primeira armadilha localizou-se a $10 \mathrm{~m}$ da borda, a segunda a 50m da borda e a terceira a 100 da borda, em cada área, totalizando seis armadilhas (Fig. 1). A posição das armadilhas, bem como as principais características florísticas de cada um dos pontos, para as quais se contou com a colaboração de Alexandre Koehler, do Centro de Ciências Agrárias e Ambientais, Pontifícia Universidade Católica do Paraná, são descritas abaixo, com as abreviaturas dos pontos, que serão utilizadas no restante desta publicação:

Ponto 1 (P100) - (2557'31,527"S, 49¹5'2,573"W). A armadilha malaise foi instalada a $100 \mathrm{~m}$ da borda de contato dos dois ambientes, a 952 m s.n.m., na extremidade do transecto. Neste ponto, o mais interior na plantação de Pinus elliottii, os exemplares atingem uma altura média de $18 \mathrm{~m}$. O solo é coberto por acículas de onde surge um pequeno número de espécies vegetais de pequeno porte, representadas por: Leandra regnellii Cogn. (Melastomataceae); Centella asiatica (Linnaeus) Urban (Apiaceae); Coccocypselum lanceolatum Pers. (Rubiaceae).

Ponto 2 (P50) - A armadilha instalada a $50 \mathrm{~m}$ da borda, a 960 m s.n.m., dentro do plantio de Pinus elliottiii, com altura média em torno de $18 \mathrm{~m}$. No sub-bosque, entre as espécies que compõe a regeneração natural, destacam-se o Machaerium stipitatum Vogel (Fabaceae); Cedrela fissilis Vell. (Meliaceae); e Dendropanax sp. (Araliaceae). O solo é coberto por acículas com algumas espécies vegetais, predominando: Leandra regnellii; Piper gaudichaudianum Kunth (Piperaceae); e Rumohra adiantiformis (G. Forest.) Ching (Dryopteridaceae).

Ponto 3 (PB) - A armadilha foi instalada a dez metros do início da plantação de Pinus elliottii, a 974 m s.n.m. O plantio de Pinus elliottiii não é tão denso quanto nos dois pontos anteriores e apresentam altura média de $16 \mathrm{~m}$. O sub-bosque é mais denso, em função da maior entrada de luz no solo da floresta, este também coberto por acículas. As espécies que compõem a regeneração natural neste estrato são: Lithraea brasiliensis March. (Anacardiaceae) acompanhada dePiper sp. (Piperaceae); Cupania vernalis Cambess. (Sapindaceae); eZanthoxylum rhoifolium Lam. (Rutaceae). As espécies vegetais que predominam no solo são: Leandra regnellii; Piper gaudichaudianum; e Geonoma schottiana Mart. (Arecaceae).

Ponto 4 (FB) - Armadilha instalada a $10 \mathrm{~m}$ da borda de contato dos ambientes, dentro da Floresta Ombrófila Mista, a 980 m s.n.m. Encontra-se em estágio médio de sucessão, com fisionomia de capoeirão. O estrato arbóreo é composto por Cabralea canjerana (Vell.) Mart. (Meliaceae); Zanthoxylum rhoifolium; Myrsine ferruginea Spreng.(Myrsinaceae); Myrsine umbellata Mart. (Myrsinaceae); Casearia sylvestris Sw. (Flacourtiaceae); Symplocos uniflora Benth. (Symplocaceae); Nectandra lanceolata Nees \& Mart.(Lauraceae); Cedrela fissilis; Syagrus romanzoffiana (Cham.) Glassm. (Palmae); Lamanonia speciosa (Cambess.) L. B. Sm. (Cunoniaceae); e Clethra scabra Pers. (Clethraceae). O sub-bosque é composto de espécies arbóreas, ainda juvenis, como Cupania vernalis; M aytenus alaternoides Reissek (Celastraceae); Symplocos sp. (Symplocaceae); e Schinus

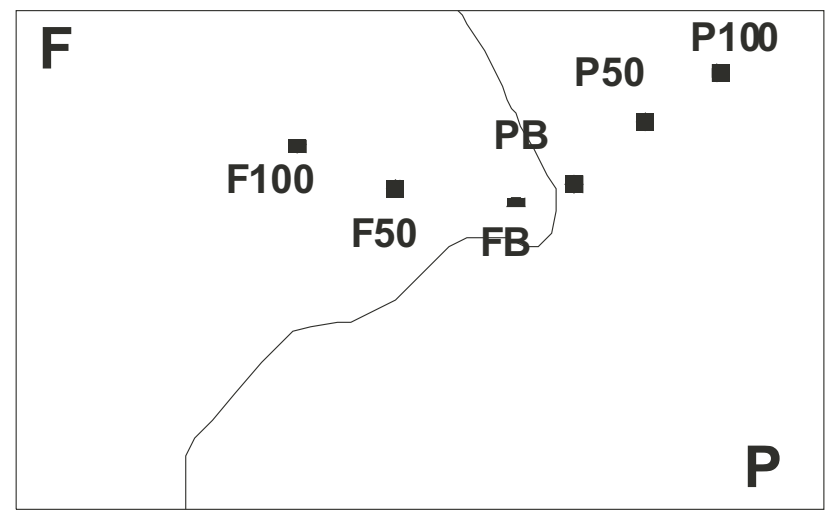

Figura 1. Posição das armadilhas malaise nas duas áreas, floresta natural (Floresta Ombrófila Mista) (F) e plantação de Pinus elliotti (P), dentro do Parque Ecológico Vivat Floresta, Tijucas do Sul, Paraná. (B) borda, (50) 50 m da borda, (100) 100 m da borda.

terebinthifolius Raddi (Anacardiaceae). As espécies vegetais que predominam no solo são: Coccocypselum lanceolatum; Rubus erythrocladus Mart (Rosaceae); Ichnanthus pallens (Sw) (Poaceae); e Axonopus compressus (Swartz) P. Beauv. (Poaceae).

Ponto 5 (F50) - Armadilha instalada a $50 \mathrm{~m}$ da borda de contato, a 996 m s.n.m. Neste local a floresta apresenta característica fisionômicas e de sucessão semelhante ao anterior. Predominam Inga edulis Mart. (Fabaceae); Zanthoxylum rhoifolium; Alchornea triplinervia Müll. Arg. (Euphorbiaceae); e Myrsine umbellata. No estrato abaixo predominam a Clethra scabra; Cupania vernalis; Caseria sylvestris; Dalbergia brasiliensis Vogel (Fabaceae); além da Sebastiana brasiliensis (Spreng) Müll. Arg. (Euphorbiaceae). Ocorre ainda uma Asteraceae arbórea que não ultrapassa os $4 \mathrm{~m}$ de altura. No solo predominam as espécies: Rubus erythrocladus; Mikania glomerata Spreng (Asteraceae) e Mikania sp. (Asteraceae); Ichnanthus tenuis (Presl.) Hitchc \& Chase (Poaceae); Leandra regnellii; Anemia phyllitidis (L.) (Schizaeaceae); Ctenitis falciculata (Raddi) Ching (Dryopteridaceae).

Ponto 6 (F100) - (2557' 32,533"S, 49 15' 10,467"W). Armadilha instalada a $100 \mathrm{~m}$ do ponto de contato, a $1011 \mathrm{~m}$ s.n.m. Neste local a Floresta Ombrófila Mista encontra-se no mais avançado estágio de desenvolvimento, comparada aos demais locais de amostragem, e descrita como capoeirão. No dossel predominam Cupania vernalis; Caseria sylvestris; Myrsine umbellata; Lamanonia speciosa; Clethra scabra; Laplacea fruticosa (Schrad.) Kobuski (Theaceae); e Casearia obliqua Spreng. (Flacourtiaceae). No segundo estrato aparecem com freqüência Gordonia fruticosa (Schrad.) H. Keng (Theaceae); Clethra scabra; e Cupania vernalis. Chamam atenção espécies da família Monimiaceae (Mollinedia spp.), ocupando o estrato arbustivo. As espécies vegetais que predominam no solo são: Miconia theazans (Bompl.) Cogn. (M elastomataceae); Ichnanthus tenuis; Cupania vernalis; Leandra regnellii; Piper gaudichaudianum; e Rumohra adiantiformis. 


\section{Método de coleta}

As armadilhas malaise foram instaladas dispondo o maior eixo no sentido Norte-Sul, com o frasco coletor voltado para o Norte, aproveitando o comportamento dos insetos que, uma vez interceptados, tendem a subir, e são conduzidos ao recipiente coletor que contém álcool $70 \%$ como conservante.

Observando os ciclos sazonais comuns às áreas temperadas (W OLDA 1988, SUTHERST et al. 1995), a coleta foi realizada por 12 meses, de agosto de 2004 a julho de 2005. O material foi retirado do frasco coletor, semanalmente, às sextas-feiras à tarde, totalizando 52 amostras.

\section{Material e identificação}

Os Coleoptera foram identificados ao nível de família, seguindo a classificação proposta por LAWRENCE \& NEWTON (1995), com as modificações anotadas em LAWRENCE et al. (2000). A identificação dos Curculionoidea ao nível de família seguiu a classificação proposta por Wibmer \& O’Brien (1986, 1989). Para a identificação foram utilizadas as chaves dicotômicas e/ou caracterizações contidas na literatura taxonômica (ARNETt 1968, Golbach 1994, Lawrence \& Britton 1991, Lawrence et al. 2000, LIMA 1952-56), bem como comparando ao material existente na Coleção de Entomologia Pe Jesus Santiago Moure. Para facilitar o acesso às informações, os exemplares coletados foram registrados em um banco de dados relacional gerenciado pelo programa BONABIO, em base MS Access 2000, desenvolvido por S.R. Bonatto (UFPR). O material coletado está depositado na Coleção de Entomologia Pe. Jesus Santiago Moure.

\section{Análises estatísticas e de agrupamento}

As análises estatísticas foram feitas pelo programa Statistica 6. StatSoft, Inc. (2001), e as de agrupamento foram feitas utilizando o programa NTSYS-pc (Numerical Taxonomy and MultivariateAnalysis System), versão 2.02, desenvolvido por RoHLF (1989), com o objetivo de identificar as relações entre as localidades amostradas; os métodos empregados são indicados no texto.

\section{RESULTADOS}

\begin{abstract}
Abundância e riqueza
Nos seis pontos de coleta foram capturados 12397 exemplares. A abundância foi significativamente maior (ANOVA $p<$ 10) na área de floresta natural (7204) e menor na área de plantação de pinus (5193). O maior número de indivíduos (2697) foi capturado no ponto F100, na floresta natural, e o menor número (1323), no ponto P100 na plantação de pinus, localizados em extremos opostos (Tab. I). Os valores de abundância foram crescentes, do ponto extremo do transecto na plantação de pinus ao ponto extremo da floresta natural. Teste designificância (ANOVA $p<10$ ) indicou diferença significativa de abundância somente entre os pontos extremos P100 x F100 (Tab. II). Considerando apenas os três pontos da floresta nativa, houve um aumento de abundância da borda para o ponto F100; enquanto que no pinus houve um decréscimo da borda para o ponto P100.
\end{abstract}

Tabela I. Coleópteros capturados nos seis pontos inventariados no Parque Ecológico Vivat Floresta, Tijucas do Sul, Paraná, durante o período de agosto de 2004 a julho de 2005. (F) Floresta natural (Floresta Ombrófila Mista), (P) plantação de Pinus elliotti, (B) borda, (50) $50 \mathrm{~m}$ da borda, (100) $100 \mathrm{~m}$ da borda.

\begin{tabular}{|c|c|c|c|c|c|c|}
\hline & \multicolumn{2}{|c|}{ Pinus } & \multicolumn{3}{|c|}{ Floresta natural } & \multirow{2}{*}{ Total } \\
\hline & P100 P50 & PB & FB & F50 & F100 & \\
\hline $\mathrm{N}^{\circ}$ de indivíduos & 13231863 & 2007 & 2011 & 2496 & 2697 & 12397 \\
\hline
\end{tabular}

Tabela II. Valores de "p" a partir dos dados de abundância dos coleópteros capturados nos seis pontos estudados no Parque Ecológico Vivat Floresta, Tijucas do Sul, Paraná, durante o período de agosto de 2004 a julho de 2005. Valor em negrito indica diferença significativa $(p<0,10)$. (F) Floresta natural (Floresta Ombrófila Mista), (P) plantação de Pinus elliotti, (B) borda, (50) 50 m da borda, (100 ) $100 \mathrm{~m}$ da borda.

\begin{tabular}{lcccccc}
\hline Pontos & P100 & P50 & PB & FB & F50 & F100 \\
\hline P100 & X & & & & & \\
P50 & 0,306 & X & & & & \\
PB & 0,218 & 0,814 & X & & & \\
FB & 0,231 & 0,814 & 0,995 & $X$ & & \\
F50 & 0,117 & 0,424 & 0,546 & 0,555 & X & \\
F100 & $\mathbf{0 , 0 7 3}$ & 0,301 & 0,402 & 0,412 & 0,834 & X \\
\hline
\end{tabular}

Nas duas áreas foram capturadas 57 famílias (Tab. III), sendo 54 na plantação de pinus e 53 na floresta natural. Quarenta famílias ocorreram em todos os pontos amostrados, enquanto que a maioria das 17 restantes ocorreu em apenas um ou dois pontos, através de um ou dois indivíduos por ponto, como Aderidae, Belidae, Brentidae, Ceratocanthidae, Clambidae, Colydiidae, Cryptophagidae, Dermestidae, Hydrophilidae, Megalopodidae, Scydmaenidae, entre outras. Destas, quatro foram encontradas apenasna plantação de pinus (Aderidae, Clambidae, Corylophidae e Hydrophilidae); e três na floresta natural (Ceratocanthidae, Dermestidae e Megalopodidae). A maior riqueza foi encontrada no ponto $\mathrm{PB}$, onde foram capturadas 50 famílias; o menor número, 42, foi observado nos pontos P50 e F50. Um total de 35 famílias foi comum a todos os pontos, enquanto 12 famílias foram capturadas em apenas um ou dois dos seis pontos.

Relação entre as áreas a partir dos dados de abundância de cada um dos pontos de inventariamento

Conforme indicado pela árvore de agrupamento, obtida por UPGMA, a partir de matriz de semelhança pelo coeficiente de correlação linear (Fig. 2), há uma maior relação de semeIhança entre os pontos de inventariamento mais próximos, que se destaca pela semelhança entre os pontos de borda (PB x FB); ao mesmo tempo que evidencia a diferença de fauna entre os dois ambientes, plantação de pinus e floresta natural, pelos pontos extremos do transecto (P100+P50 x F50+F100). 


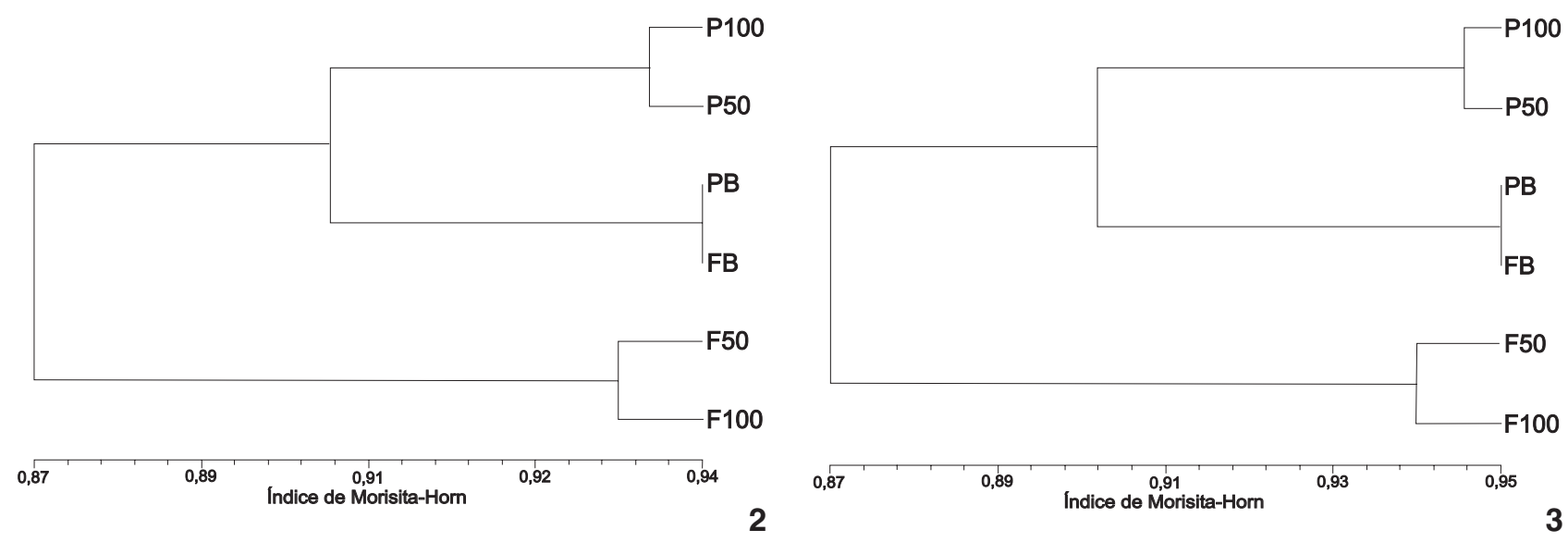

Figuras 2-3. Análise de agrupamento das famílias de Coleoptera capturadas durante o período de agosto de 2004 a julho de 2005 , no Parque Ecológico Vivat Floresta Tijucas do Sul, Paraná. (2) Pontos inventariados x dados de abundância de todas as famílias; (3) Pontos inventariados $x$ dados de abundância das famílias dominantes incluídas em $60 \%$ do total da abundância. (F) Floresta Ombrófila Mista, (P) plantação de Pinus elliotti, (B) borda, (50) 50 m da borda, (100) 100 m da borda.

Tabela III. Abundância e percentuais de famílias de Coleoptera capturadas com malaise, em seis pontos, no Parque Ecológico Vivat Floresta, Tijucas do Sul, Paraná, durante o período de agosto de 2004 a julho de 2005.

\begin{tabular}{|c|c|c|c|c|c|c|c|c|c|c|c|c|c|c|c|}
\hline \multirow{2}{*}{ Famílias } & \multicolumn{6}{|c|}{ Plantação de Pinus elliotti } & \multicolumn{6}{|c|}{ Floresta natural (Floresta Ombrófila Mista) } & \multirow{2}{*}{ Total } & \multirow{2}{*}{$\%$} & \multirow{2}{*}{$60 \%$} \\
\hline & $100 \mathrm{~m}$ & $50 \mathrm{~m}$ & Borda & Total & $\%$ & $60 \%$ & Borda & $50 \mathrm{~m} 1$ & $00 \mathrm{~m}$ & Total & $\%$ & $60 \%$ & & & \\
\hline Aderidae & 2 & 0 & 2 & 4 & 0,08 & & 0 & 0 & 0 & 0 & 0,00 & & 4 & 0,03 & \\
\hline Anobiidae & 5 & 5 & 8 & 18 & 0,35 & & 5 & 2 & 8 & 15 & 0,21 & & 33 & 0,27 & \\
\hline Anthicidae & 0 & 2 & 4 & 6 & 0,12 & & 1 & 0 & 0 & 1 & 0,01 & & 7 & 0,06 & \\
\hline Anthribidae & 10 & 7 & 33 & 50 & 0,96 & & 13 & 8 & 12 & 33 & 0,46 & & 83 & 0,67 & \\
\hline Apionidae & 2 & 1 & 2 & 5 & 0,10 & & 1 & 1 & 3 & 5 & 0,07 & & 10 & 0,08 & \\
\hline Belidae & 0 & 0 & 1 & 1 & 0,02 & & 1 & 0 & 0 & 1 & 0,01 & & 2 & 0,02 & \\
\hline Bostrichidae & 0 & 0 & 4 & 4 & 0,08 & & 0 & 1 & 0 & 1 & 0,01 & & 5 & 0,04 & \\
\hline Brentidae & 1 & 0 & 0 & 1 & 0,02 & & 0 & 0 & 1 & 1 & 0,01 & & 2 & 0,02 & \\
\hline Buprestidae & 2 & 8 & 5 & 15 & 0,29 & & 13 & 26 & 9 & 48 & 0,67 & & 63 & 0,51 & \\
\hline Cantharidae & 11 & 20 & 30 & 61 & 1,17 & & 27 & 53 & 62 & 142 & 1,97 & & 203 & 1,64 & \\
\hline Carabidae & 15 & 24 & 27 & 66 & 1,27 & & 37 & 15 & 31 & 83 & 1,15 & & 149 & 1,20 & \\
\hline Cerambycidae & 219 & 171 & 129 & 519 & 9,99 & 10,0 & 137 & 368 & 407 & 912 & 12,66 & 12,7 & 1431 & 11,54 & 11,5 \\
\hline Ceratocanthidae & 0 & 0 & 0 & 0 & 0,00 & & 1 & 0 & 0 & 1 & 0,01 & & 1 & 0,01 & \\
\hline Chenolariidae & 2 & 4 & 11 & 17 & 0,33 & & 6 & 5 & 7 & 18 & 0,25 & & 35 & 0,28 & \\
\hline Chrysomelidae & 95 & 102 & 175 & 372 & 7,16 & 7,2 & 202 & 423 & 392 & 1017 & 14,12 & 14,1 & 1389 & 11,20 & 11,2 \\
\hline Clambidae & 0 & 0 & 3 & 3 & 0,06 & & 0 & 0 & 0 & 0 & 0,00 & & 3 & 0,02 & \\
\hline Cleridae & 28 & 57 & 62 & 147 & 2,83 & & 36 & 54 & 38 & 128 & 1,78 & & 275 & 2,22 & \\
\hline Coccinellidae & 44 & 40 & 67 & 151 & 2,91 & & 102 & 199 & 59 & 360 & 5,00 & & 511 & 4,12 & \\
\hline Colydiidae & 4 & 0 & 0 & 4 & 0,08 & & 0 & 0 & 4 & 4 & 0,06 & & 8 & 0,06 & \\
\hline Corylophidae & 3 & 1 & 2 & 6 & 0,12 & & 0 & 0 & 0 & 0 & 0,00 & & 6 & 0,05 & \\
\hline Cryptophagidae & 0 & 1 & 0 & 1 & 0,02 & & 1 & 0 & 0 & 1 & 0,01 & & 2 & 0,02 & \\
\hline Cucujidae & 5 & 15 & 10 & 30 & 0,58 & & 7 & 12 & 2 & 21 & 0,29 & & 51 & 0,41 & \\
\hline Curculionidae & 105 & 161 & 229 & 495 & 9,53 & 9,5 & 269 & 221 & 214 & 704 & 9,77 & 9,8 & 1199 & 9,67 & 9,7 \\
\hline Dascillidae & 1 & 0 & 2 & 3 & 0,06 & & 1 & 0 & 0 & 1 & 0,01 & & 4 & 0,03 & \\
\hline
\end{tabular}


Tabela III. Continuação.

\begin{tabular}{|c|c|c|c|c|c|c|c|c|c|c|c|c|c|c|c|}
\hline \multirow{2}{*}{ Famílias } & \multicolumn{6}{|c|}{ Plantação de Pinus elliotti } & \multicolumn{6}{|c|}{ Floresta natural (Floresta Ombrófila Mista) } & \multirow{2}{*}{ Total } & \multirow{2}{*}{$\%$} & \multirow{2}{*}{$60 \%$} \\
\hline & $100 \mathrm{~m}$ & $50 \mathrm{~m}$ & Borda & Total & $\%$ & $60 \%$ & Borda & $50 \mathrm{~m}$ & $100 \mathrm{~m}$ & Total & $\%$ & $60 \%$ & & & \\
\hline Dermestidae & 0 & 0 & 0 & 0 & 0,00 & & 0 & 0 & 1 & 1 & 0,01 & & 1 & 0,01 & \\
\hline Elateridae & 37 & 67 & 66 & 170 & 3,27 & & 133 & 85 & 167 & 385 & 5,34 & & 555 & 4,48 & \\
\hline Endomychidae & 38 & 37 & 39 & 114 & 2,20 & & 25 & 25 & 20 & 70 & 0,97 & & 184 & 1,48 & \\
\hline Erotylidae & 22 & 42 & 18 & 82 & 1,58 & & 9 & 8 & 16 & 33 & 0,46 & & 115 & 0,93 & \\
\hline Eucnemidae & 13 & 36 & 23 & 72 & 1,39 & & 48 & 36 & 19 & 103 & 1,43 & & 175 & 1,41 & \\
\hline Geotrupidae & 1 & 0 & 2 & 3 & 0,06 & & 1 & 2 & 2 & 5 & 0,07 & & 8 & 0,06 & \\
\hline Hydrophilidae & 0 & 0 & 1 & 1 & 0,02 & & 0 & 0 & 0 & 0 & 0,00 & & 1 & 0,01 & \\
\hline Lampyridae & 123 & 157 & 155 & 435 & 8,38 & 8,4 & 117 & 262 & 185 & 564 & 7,83 & 7,8 & 999 & 8,06 & 8,1 \\
\hline Lathridiidae & 3 & 1 & 1 & 5 & 0,10 & & 1 & 0 & 0 & 1 & 0,01 & & 6 & 0,05 & \\
\hline Leiodidae & 11 & 20 & 13 & 44 & 0,85 & & 2 & 3 & 13 & 18 & 0,25 & & 62 & 0,50 & \\
\hline Lucanidae & 1 & 0 & 1 & 2 & 0,04 & & 1 & 0 & 0 & 1 & 0,01 & & 3 & 0,02 & \\
\hline Lycidae & 6 & 11 & 23 & 40 & 0,77 & & 9 & 16 & 14 & 39 & 0,54 & & 79 & 0,64 & \\
\hline Megalopodidae & 0 & 0 & 0 & 0 & 0,00 & & 0 & 1 & 0 & 1 & 0,01 & & 1 & 0,01 & \\
\hline Melandryidae & 7 & 7 & 1 & 15 & 0,29 & & 1 & 4 & 3 & 8 & 0,11 & & 23 & 0,19 & \\
\hline Monommatidae & 1 & 3 & 3 & 7 & 0,13 & & 6 & 1 & 1 & 8 & 0,11 & & 15 & 0,12 & \\
\hline Mordelidae & 49 & 105 & 117 & 271 & 5,22 & & 134 & 124 & 97 & 355 & 4,93 & & 626 & 5,05 & \\
\hline Mycetophagidae & 6 & 2 & 2 & 10 & 0,19 & & 1 & 5 & 0 & 6 & 0,08 & & 16 & 0,13 & \\
\hline Mycteridae & 0 & 4 & 1 & 5 & 0,10 & & 3 & 4 & 3 & 10 & 0,14 & & 15 & 0,12 & \\
\hline Nitidulidae & 114 & 227 & 147 & 488 & 9,40 & 9,4 & 223 & 87 & 240 & 550 & 7,63 & 7,6 & 1038 & 8,37 & 8,4 \\
\hline Oedemeridae & 9 & 15 & 16 & 40 & 0,77 & & 14 & 12 & 1 & 27 & 0,37 & & 67 & 0,54 & \\
\hline Phalacridae & 1 & 6 & 1 & 8 & 0,15 & & 3 & 1 & 2 & 6 & 0,08 & & 14 & 0,11 & \\
\hline Phengodidae & 16 & 43 & 30 & 89 & 1,71 & & 15 & 60 & 36 & 111 & 1,54 & & 200 & 1,61 & \\
\hline Platypodidae & 1 & 4 & 2 & 7 & 0,13 & & 7 & 1 & 10 & 18 & 0,25 & & 25 & 0,20 & \\
\hline Ptilodactylidae & 38 & 88 & 82 & 208 & 4,01 & & 83 & 74 & 94 & 251 & 3,48 & & 459 & 3,70 & \\
\hline Scarabaeidae & 7 & 4 & 6 & 17 & 0,33 & & 13 & 7 & 22 & 42 & 0,58 & & 59 & 0,48 & \\
\hline Scirtidae & 1 & 1 & 7 & 9 & 0,17 & & 11 & 4 & 12 & 27 & 0,37 & & 36 & 0,29 & \\
\hline Scolytidae & 125 & 156 & 125 & 406 & 7,82 & 7,8 & 116 & 126 & 134 & 376 & 5,22 & & 782 & 6,31 & 6,3 \\
\hline Scraptiidae & 6 & 3 & 8 & 17 & 0,33 & & 3 & 3 & 35 & 41 & 0,57 & & 58 & 0,47 & \\
\hline Scydmaenidae & 2 & 0 & 0 & 2 & 0,04 & & 0 & 0 & 1 & 1 & 0,01 & & 3 & 0,02 & \\
\hline Silvanidae & 6 & 5 & 12 & 23 & 0,44 & & 4 & 4 & 16 & 24 & 0,33 & & 47 & 0,38 & \\
\hline Staphylinidae & 90 & 159 & 255 & 504 & 9,71 & 9,7 & 139 & 115 & 221 & 475 & 6,59 & 6,6 & 979 & 7,90 & 7,9 \\
\hline Tenebrionidae & 33 & 41 & 42 & 116 & 2,23 & & 29 & 37 & 80 & 146 & 2,03 & & 262 & 2,11 & \\
\hline Trogossitidae & 2 & 0 & 2 & 4 & 0,08 & & 0 & 1 & 3 & 4 & 0,06 & & 8 & 0,06 & \\
\hline Total indivíduos & 1323 & 1863 & 2007 & 5193 & 100,0 & 62,0 & 2011 & 2496 & 2697 & 7204 & 100,0 & 58,6 & 12397 & 100,0 & 63,1 \\
\hline Total famílias & 47 & 42 & 50 & 54 & & & 46 & 42 & 43 & 53 & & & 57 & & \\
\hline
\end{tabular}

Famílias mais abundantes

O número de famílias mais abundantes (Tab. III) envolvidas em cerca de $60 \%$ do total de indivíduos capturados no conjunto das duas áreas amostradas, foi de sete: 1) Cerambycidae, 2) Chrysomelidae, 3) Curculionidae, 4) Nitidulidae, 5) Lampyridae, 6) Staphylinidae e 7) Scolytidae. Quando observadas independentemente, as duas áreas mostraram diferenças entre as famílias dominantes. $\mathrm{Na}$ área de pinus as famílias que correspondem a cerca $60 \%$ da abundância são: 1) Cerambycidae, 2) Staphylinidae, 3) Curculionidae, 4) Nitidulidae, 5) Lampyridae 6) Scolytidae, 7) Chrysomelidae. $\mathrm{Na}$ área coberta com floresta natural as famílias dominantes foram: 1) Chrysomelidae, 2) Cerambycidae, 3) Curculionidae, 4) Lampyridae, 5) Nitidulidae, 6) Staphylinidae. Nesta área apenas seis famíliasjá perfazem um percentual (58,6\%), mostrando ser Elateridae a sétima família dentre as mais abundantes, mas que elevaria o percentual para $63,9 \%$. Conforme se 
observa na tabela, há apenas uma família que não consta entre as mais abundantes nas duas áreas, Scolytidae, relacionada apenas na área de pinus. As famílias Cerambycidae, Curculionidae, Lampyridae e Nitidulidae apresentam posição de dominância assemelhadas nas duas áreas. A família Chrysomelidae, no entanto, foi muito abundante na área de floresta nativa, atingindo 14,1\% da abundância total da área. Na área de pinus esta família foi percentualmente menos expressiva, correspondendo a 7,2\% do total. O oposto ocorreu com Staphylinidae, que na floresta natural representou apenas $6,6 \%$ da abundancia total, eem pinus foi o segundo maior valor percentual $(9,7 \%)$.

Analisando individualmente cada ponto amostrado, cinco a sete famílias compreendem cerca de $60 \%$ da abundância total de cada área (Tab. IV). Nos pontos P50 e PB da plantação de pinus foram anotadas sete famílias para atingir $60 \%$; enquanto que no ponto $\mathrm{P} 100$ foram apenas seis famílias. Na floresta natural foram sete famílias no ponto FB; cinco famílias em F50 e seis em F100. Algumas famílias mais abundantes em al guns dos pontos não aparecem como tal no conjunto das áreas, como visto acima. Assim foram Mordelidae, no ponto P50; Mordelidae e Elateridae, no ponto FB; Coccinellidae, no ponto F50.

Na plantação de pinus, as famílias mais abundantes são as mesmas em todos os pontos, exceto no ponto P50, onde Mordelidae é mais abundante que Chrysomelidae. Na floresta natural apenas três famílias são comuns aos três pontos: Curculionidae, Chrysomelidae e Cerambycidae.

As relações entre os pontos de coleta (Fig. 3), analisados pela árvore de agrupamento, a partir de dados de abundância das famílias que são incluídas dentro dos $60 \%$ do total da abundância, indica a proximidade maior entre os pontos de plantação de pinus (P100 x P50), aos quais se une o grupo formado pelos pontos do ecótono ( $\mathrm{PB} \times \mathrm{FB}$ ); e a estes o grupo que inclui os dois pontos extremos da floresta natural.

\section{DISCUSSÃO}

Abundância

A floresta natural apresentou maior abundância de besouros que a plantação de pinus. Houve uma maior semelhança das comunidades de Coleoptera entre os pontos de cada uma das áreas, caracterizando as diferenças florísticas e estruturais da plantação de pinus com a de Floresta Ombrófila Mista. A abundância foi crescente do ponto mais interior da plantação de pinus para o interior da floresta natural; possivelmente relacionada à maior diversidade florística e à possível maior disponibilidade trófica desta última área, como indicam as características vegetais dos diferentes pontos, descritas na metodologia. Levandose em consideração as abundâncias totais dos pontos amostrados na área de floresta natural em Tijucas do Sul, observamos a tendência de aumento nas áreas mais diversificadas florísticamente. Nos inventariamentos do PROFAUPAR (M ARINONI \& DUTRA 1997), nas localidades situadas no interior da região de domínio da Floresta Ombrófila Mista, com uso de armadilha malaise, também foi registrado este fato; Colombo, localidade florísticamente muito alterada, comparável a estágio inicial de sucessão vegetal, apresentou menor abundância do que aquela considerada em estágio mais avançado, Ponta Grossa (Vila Velha). No entanto, em áreas com diferentes estágios de sucessão vegetal, nos inventariamentos do PROVIVE (GANHO \& M ARINONI 2003), foram capturados mais indivíduos nas áreas em estágios inicial/intermediário de sucessão vegetal que na mais avançada. O ponto onde se deu a coleta na área considerada em estágio intermediário de sucessão vegetal durante o PROVIVE (1999-2000) foi o mesmo onde se deu a coleta pelo PROFAUPAR (1986-1987).

\section{Riqueza de famílias}

Nas duas áreas foi coletado praticamente o mesmo número de famílias, diferindo pela presença de poucas e raras famílias. Em Tijucas do Sul, assim como em Vila Velha, a riqueza de famílias em cada ponto amostrado não evidenciou uma re lação com a abundância observada e nem com o nível de sucessão das mesmas. Em Tijucas do Sul, as maiores riquezas se concentraram no ecótono constituído pelas bordas de contato da floresta natural e plantação de pinus. Em Vila Velha, no conjunto do inventariamento do PROVIVE foram reconhecidas mais famílias que em Tijucas do Sul, com a área de borda (floresta natural - campo), no entanto, sendo a menos rica que

Tabela IV. Famílias dominantes (cerca de $60 \%$ da abundância de cada ponto) capturadas por malaise, ordenadas pelo percentual de abundância em cada um dos seis pontos inventariados no Parque Ecológico Vivat Floresta, Tijucas do Sul, Pr, durante o período de agosto de 2004 a julho de 2005. (F) Floresta natural (Floresta Ombrófila Mista), (P) plantação de Pinus elliotti, (B) borda, (50) 50 m da borda, (100) $100 \mathrm{~m}$ da borda.

\begin{tabular}{|c|c|c|c|c|c|c|c|}
\hline P100 & $\%$ & P50 & PB & FB & F50 & F100 & $\%$ \\
\hline Cerambycidae & 16,55 & Nitidulidae & 12,18 Staphylinidae & 12,71 Curculionidae & 13,38 Chrysomelidae & 16,95 Cerambycidae & 15,09 \\
\hline Scolytidae & 9,45 & Cerambycidae & 9,18 Curculionidae & 11,41 Nitidulidae & 11,09 Cerambycidae & 14,74 Chrysomelidae & 14,53 \\
\hline Lampyridae & 9,30 & Curculionidae & 8,64 Chrysomelidae & 8,72 Chrysomelidae & 10,04 Lampyridae & 10,50 Nitidulidae & 8,90 \\
\hline Nitidulidae & 8,62 & Staphylinidae & 8,53 Lampyridae & 7,72 Staphylinidae & 6,91 Curculionidae & 8,85 Staphylinidae & 8,19 \\
\hline Curculionidae & 7,94 & Lampyridae & 8,43 Nitidulidae & 7,32 Cerambycidae & 6,81 Coccinellidae & 7,97 Curculionidae & 7,93 \\
\hline \multirow[t]{3}{*}{ Chrysomelidae } & 7,18 & Scolytidae & 8,37 Cerambycidae & 6,43 Mordelidae & 6,66 & Lampyridae & 6,86 \\
\hline & & Mordelidae & 5,64 Scolytidae & 6,23 Elateridae & 6,61 & & \\
\hline & 59,04 & & 60,97 & 60,54 & 61,50 & 59,01 & 61,50 \\
\hline
\end{tabular}


as áreas do interior da floresta. Os diferentes tipos de ecótonos representados por floresta-floresta e floresta-campo talvez possam explicar a maior riqueza deste ponto em Tijucas do Sul. Comparando as famílias capturadas na área de floresta natural de Tijucas do Sul com os dados obtidos nas três áreas em sucessão vegetal de Vila Velha observa-se que quatro famílias foram encontradas apenas em Tijucas do Sul: Colydiidae, Cryptophagidae, Dascillidae e Lucanidae; enquanto que as famílias Attelabidae, Clambidae, Corylophidae, Dytiscidae, Eucinetidae, Histeridae, Hydrophilidae, Languridae, Melyridae, e Rhipiphoridae foram capturadas apenas em Vila Velha. A maior riqueza em Vila Velha ficando assim, caracterizada provavelmente pela existência das diferentes fases de sucessão vegetal inventariadas.

Não foi possível encontrar na literatura trabalhos que se referissem a levantamentos de Coleoptera capturados por armadilha malaise em ambientes assemelhados aos do presente trabalho. Em inventário na Malásia, CHung et al. (2000) utilizaram vários tipos de armadilhas em diferentes ambientes. Um dos ambientes era uma floresta natural manejada, com a retirada seletiva de árvores, e outro era uma plantação de acácia, exótica. Na armadilha de interceptação de vôo, com uma área de $2,5 \mathrm{~m}^{2}$, que é a que tem características mais próximas às de malaise, tanto a abundância de indivíduos como o número de famílias foram superiores na floresta manejada. Em Tijucas do Sul, apesar de ter havido a mesma relação, as diferenças na abundância e na riqueza de famílias entre as áreas foram muito menores. HutCHESON \& Jones (1999), avaliando a fauna de Insecta, através de espécies de Coleoptera, em plantação exótica de Pinus radiata, encontrou um alto índice de endemicidae; considerou ser possível que plantações exóticas economicamente produtivas possam sustentar uma biodiversidade endêmica. Em Tijucas do Sul, em que se analisa a diversidade de famílias em áreas de plantação exótica e de floresta natural foi possível verificar a existência de cerca de $90 \%$ de famílias que são comuns a ambas. Consi derando que a flora rasteira e do sub-bosque da plantação de pinus é muito mais pobre que a da floresta natural e não há a penetração de nenhuma planta nativa no dossel, a semelhança observada entre as famílias pode se repetir quando forem feitas análises com as espécies (trabalho em andamento). Este fato configuraria a existência da semelhança em Tijucas do Sul como uma conseqüência da fauna da plantação de pinus ser vagrante, oriunda da floresta natural.

As análises com dados de totais de riqueza de famílias devem ser consideradas com certa reserva. O número de famílias nos diferentes inventariamentos mostra pequenas variações. Estas são decorrentes principalmente da presença/ausência de famílias consideradas como raras. Na comparação entre diferentes publicações, além deste fator deve-se considerar que alterações de categoria taxonômica, com subfamília sendo elevada à categoria de família e vice-versa como decorrência de estudos sistemáticos que se processam ao longo dos anos, se refletem em diferentes valores de riqueza, afetando as relações.

\section{Famílias mais abundantes}

Dentre as sete famílias mais abundantes, considerando as duas áreas amostradas, verifica-se que Cerambycidae, Curculionidae, Lampyridae e Nitidulidae apresentaram posições de dominância assemelhadas. Diferentemente, Chrysomelidae, Staphylinidae e Scolytidae apresentaram níveis de dominância desiguais. Chrysomelidae foi mais abundante na área de floresta nativa onde havia uma presença maior de plantas rasteiras e arbustivas com maior disponibilidade de folhas sazonais, o que não foi observado na área de pinus. A família Staphylinidae, com a maioria das espécies tendo hábitos carnívoros e detritívoros, foi por outro lado das mais abundantes na plantação de pinus.

A análise de agrupamento das relações entre os diferentes pontos das duas áreas, a partir dos dados com abundância total comparado àquela obtida com dados de famílias que se incluem em $60 \%$ do total da abundância foi semelhante. Evidenciou-se, como já observado no estudo das famílias capturadas em Vila Velha, que a redução de 57 para 10 famílias não alterou as indicações de semel hanças entre os pontos inventariados.

O fato observado em Tijucas do Sul, de que as seis ou sete famílias mais abundantes perfazem cerca de $60 \%$ da abundância dos coleópteros em cada área, tem se mostrado recorrente. Da mesma forma, as análises comparativas das composições das comunidades de Coleoptera entre áreas, apoiadas em dados totais de abundância, têm apresentado resultados semeIhantes àqueles produzidos a partir de análises com apenas as famílias dominantes, classificadas dentre os primeiros $60 \%$ da abundância total de cada área (MARINONI \& DutRA 1997, GANHO \& MARINONI 2003). A possibilidade do emprego desta característica de composição da abundância de Coleoptera através do uso de apenas seis a sete famílias de cada área, incluindo $60 \%$ do total do material inventariado, para se estabelecerem comparações entre as comunidades de diferentes pontos, poderá facilitar de maneira significativa os estudos sobre as condições ambientais e de similitude de áreas florestadas.

\section{AGRADECIMENTOS}

Aos pesquisadores Germano Rosado Neto, Adelita Linzmeier e Edílson Caron (UFPR), pelo auxílio na identificação de coleópteros; Alexandre Koehler (PUCPR), pela análise da situação florística e identificação dos estágios sucessionais das áreas estudadas, bem como a Armando Cervi (UFPR), pela identificação de numerosas espécies vegetais; Instituição Filantrópica Sergius Erdlyi e Pontifícia Universidade Católica do Paraná por permitir a coleta no Parque Ecológico Vivat Floresta e por propiciar condições de trabalho através do auxílio de Luiz Carlos Oliveira Santos; IBAMA pela autorização de coleta; CNPq pela concessão de bolsas e auxílio financeiro.

\section{REFERÊNCIAS BIBLIOGRÁFICAS}

Altieri, M.A; E.N. Silva \& C.I. Nichols. 2003. O papel da biodiversidade no manejo de pragas. Ribeirão Preto, Editora Holos, 226p. 
Arnet, R.H. 1968. The Beetles of the United States. A manual for identification. Washington, American Entomological Institute, $11122 \mathrm{p}$.

Carnus, J-M.; J. Parrotta; E.G. Brockerhoff; M. Arbez; H. Jactel; A. Kremer; D. Lamb; K. O’hara \& B. Walters. 2003. Planted Forests and Biodiversity. Disponível em: http:// www.fsanz.govt.nz/mafnet/unff-planted-forestry-meeting/ conference-papers/planted-forests-and-biodiversity.htm [acesso em 08.VI.2006].

Chung, A.Y.C.; P. Eggleton; M.R. Speight; P.M. Hammond \& V.K. CHEY. 2000. The diversity of beetle assemblages in different habitat types in Sabah, Malaysia. Bulletin of Entomological Research, Farnham Royal, 90: 475-496.

Engelmark, O.; K. Sjöberg; B. Andersson; O. Rosvall; G.I. Agren; W.L. Baker; P. Barklund; C. Björkman; D.G. Despain; B. Elfying; R.A. Ennos; M. Karlman; M.F. KNeCht; D.H. KNIGHT; N.J. Ledgard; A. LindelöW; C. Nilsson; G.F. Peterken; S. Sörlin \& M.T. SYKes. 2001. Ecological effects and management aspects of an exotic tree species: the case of lodgepole pine in Sweden. Forest Ecology and Management, Amsterdam, 141: 3-13.

FundaÇÃo SOS MatA AtLÂNTICA. 1998. Atlas da evolução dos remanescentes florestais e ecossistemas associados no Domínio da Mata Atlântica no período de 1990-1995. São Paulo, Fundação SOS Mata Atlântica. 55p.

GAnho, N.G. \& R.C. MARInoni. 2003. Fauna de Coleoptera no Parque Estadual de Vila Velha, Ponta Grossa, Paraná, Brasil. Abundância e riqueza das famílias capturadas através de armadilhas malaise. Revista Brasileira de Zoologia, Curitiba, 20 (4): 727-236.

Ganho, N.G. \& R.C. Marinoni. 2005. A diversidade inventarial de Coleoptera (Insecta) em uma paisagem antropizada do Bioma Araucária. Revista Brasileira de Entomologia, Curitiba, 49 (4): 535-543.

Golbach, R. 1994. Elateridae (Coleoptera) dela Argentina. Opera Lilloana, Tucumán, 41: 1-48.

HaRTLEY, M.J. 2002. Rationale and methods for conserving biodiversity in plantations forests. Forest Ecology and Management, Amsterdam, 155: 81-95.

Humphrey, J.W.; C. Hawes; A.J. Peace; R. Ferris-Kaan \& M.R. Jukes. 1999. Relationship between insect diversity and habitat characteristics in plantation forests. Forest Ecology and Management, Amsterdam, 113: 11-21.

Humphrey, J.W.; A.C. Newton; A.J. Peace; E. Holden. 2000. The importance of conifers plantations in northern Britain as a habitat for native fungi. Biological Conservation, Essex, 96: $241-252$.

Hutcheson, J. 1990. Characterization of terrestrial insect communities using quantified, malaise-trapped Coleoptera. Ecological Entomology, Londres, 15: 143-151.

HutCHESON, J. \& D. Jones.1999. Spatial variability of insect communities in a homogenous system: measuring biodiversity using malaise trapped beetles in a Pinus radiata plantation in New Zealand. Forest Ecology and Management, Amsterdam, 118: 93-105.

LAWRENCE, J.F. \& E.B. BrITton. 1991. Coleoptera. In: CSIRO (Ed.). The Insects of Australia. New York, Cornell University Press, vol. 2, 1137p.

LaWrence, J.F. \& A.F. Newton. 1995. Families and subfamilies de Coleoptera (With select genera, notes, references and data on family-group names), p. 779-913. In: J.F. PAPALUK \& S.A. SLIPINSKI (Eds). Biology, phylogeny, and classification of Coleoptera. Wkarsawa, Museum i Institut Zoologii PAN, 1092p.

Lawrence, J.F.; A.M. Hastings; M.J. Dallwitz; T.A. Paine \& E.J. ZURCHER. 2000. Beetles of the World. A key and information system for families and subfamilies. Canberra, CSIRO Publishing, versão 1.0, CD-ROM.

LıMA, A.M.C. 1952-56. Insetos do Brasil, Coleópteros. Rio de Janeiro, Escola Nacional de Agronomia, vols 7-10.

MARINONI, R.C. \& N.G. GANHO. 2006. A diversidade diferencial beta de Coleoptera (Insecta) em paisagem antropizada do Bioma Araucária. Revista Brasileira de Entomologia, Curitiba, 50 (1): 64-71.

Marinoni, R.C. \& R.R.C. Dutra. 1997. Famílias de Coleoptera capturadas com armadilha malaise em oito localidades do Estado do Paraná, Brasil. Diversidades alfa e beta. Revista Brasileira de Zoologia, Curitiba, 14 (3): 751-770.

Rohlf, F.J. 1989. NTSYS-PC. Numerical Taxonomy and Multivariate Analysis System. New York, Exeter Publ. Ltd, $\mathrm{VI}+187 \mathrm{p}$.

RusSEL, E.P. 1989. Enemies hypothesis: a review of the effect of vegetational diversity on predatory insects and parasitoids. Environmental Entomology, College Park, 18 (4): 590-599.

Sutherst, R.W.; G.F. MaYwald \& D.B. SkARRatT. 1995. Predicting insect distributions in a changed climate, p. 59-91. In: R. HARRINGTON \& N.E. StORK (Eds). Insects in a changing environment. Londres, Academic Press, 535p.

TownEs, H. 1972. A light-weight malaise trap. Entomological News, Philadelphia, 83: 239-247.

Vivat. 2003. Vivat Floresta Sistemas Ecológicos. Protector Naturae. Tijucas do Sul, Instituição Filantrópica Sergius Ederlyi, Sociedade Paranaense de Cultura, Pontifícia Universidade Católica do Paraná, 63p.

Wibmer, G.J. \& C.W. O’Brien. 1986. Annotated checklist of the weevils (Curculionidaesensu lato) of South America (Coleoptera: Curculionoidea). Memoirs of the American Entomological Institute, Ann Arbor, 39: I-XVI+1-563.

Wibmer, G.J. \& C.W. O'Brien. 1989. Additions and corrections to annotated checklists of the weevils of North America, Central America, and the West Indies, and of South America. Southwestern Entomologist, Dallas, 13 (Suppl.):1-49.

Wolda, H. 1988. Insect Seasonality: Why? Annual Review of Ecology and Systematic, Palo Alto, 19: 1-18.

Recebido em 28.VII.2006; aceito em 10.XI.2006.

Revista Brasileira de Zoologia 23 (4): 1159-1167, dezembro 2006 